\title{
La percepcion de la incursión de las tic en el aula desde la perspectiva de los estudiantes universitarios
}

\section{The perception of the incursion of tics in the classroom from the perspective of university students}

\author{
Byron Geovanny Hidalgo Cajo \\ Byron Fernando Castillo Parra \\ Diego Patricio Hidalgo Cajo \\ Iván Mesías Hidalgo Cajo \\ Universidad Nacional de Chimborazo, Ecuador \\ Escuela Superior Politécnica de Chimborazo, Ecuador
}

Autores para correspondencia:wgsiguenza@utpl.edu.ec, antonio.espinoza@ucuenca.edu.ec marco.guamana.@ucuenca.ec

Fecha de recepción: 28 de Noviembre 2017 - Fecha de aceptación: 15 de Junio de 2018

Resumen: Las tecnologías de la información y la comunicación (TIC) se han integrado de manera impresionante en la vida diaria, desde esta perspectiva se desea conocer su presencia e impacto en el proceso enseñanza aprendizaje. Determinar el uso y aplicación de las TIC en el aula y su influencia en el proceso enseñanza aprendizaje desde la percepción de los estudiantes y docentes universitarios de pregrado. La investigación es de tipo documental y de campo, el universo o población estuvo constituida por 82 estudiantes, y 3 docentes, la recolección de los datos fue el cuestionario. Se realizó un análisis estadístico descriptivo e inferencial Un porcentaje muy bajo de los docentes usan las TIC en el proceso enseñanza aprendizaje de la bioestadistica, sin embargo, los estudiantes creen firmemente que la incursión de las mismas dará un impacto positivo en el proceso educativo, como se ha comprobado la hipótesis planteada mediante la estadística. En la bioestadística, es poco frecuente la utilización de las TIC en el aula por lo que los estudiantes débilmente desarrollan habilidades cognitivas, la generación de ideas intuitivas a través del ensayo y error generando nuevo conocimiento. Palabras Claves: enseñanza; aprendizaje; bioestadística; docente; TIC

\begin{abstract}
Abstrac: The information and communications technology (ICT) have impressively integrated into daily life, from this perspective we want to know its presence and impact on the teaching-learning process. To determine the use and application of ICT in the classroom and their influence on the teaching-learning process from the perception of students and undergraduate university teachers. The research is documentary and field, the universe or population consisted of 82 students and 3 teachers, data collection was the questionnaire. one descriptive and inferential statistical analysis was performed A very low percentage of teachers use ICT in the teaching-learning process of biostatistics, however students strongly believe that the incursion of them will have a positive impact on the educational process as verified the hypothesis by statistical . In biostatistics, it is rare use of ICT in the classroom so that students develop cognitive skills feebly, generation of intuitive ideas through trial and error generating new knowledge.
\end{abstract}

Key Words: teaching; learning; biostatistics; teaching; ICT 


\section{Introducción}

Los estudios de investigación que se ejecutan en las aulas deben tener como objetivo principal el análisis de la existencia de la calidad educativa, y que, gracias a estas investigaciones, se consiga encontrar ventajas o desventajas que deberán ser corregidas o implementadas para la mejora continua en el proceso enseñanza aprendizaje. El desarrollo e inclusión de las TIC en la economía mundial, ha generado entornos que afectan profundamente en la sociedad, fragmentando en comunidades que se adaptan positivamente de estos recursos y aquellos que no lo hacen, situación denominada brecha digital. Es así que, en la educación no debe apartarse de esta realidad e incursionar en el uso de las tecnologías de la información y la comunicación en el aula, con docentes preparados hacia una educación moderna, de calidad y más competitiva.

"El papel que las Nuevas tecnologías pueden jugar un papel importante en el proceso enseñanza aprendizaje, por el número de sentidos que pueden estimular, y la potencialidad de los mismos en la retención de la información. Diversos estudios ya clásicos, han puesto de manifiesto como se recuerda la información siendo así que, el $10 \%$ es de lo que se ve, el $20 \%$ de los que se oye, el $50 \%$ de lo que se ve y oye, y el $80 \%$ de lo que se ve, se oye y se hace" (Cabero Almenara, 1996).

Según Hidalgo Cajo B, et al, (2017) se enfocan en la pertinencia de utilizar los recursos que las TIC ofrece como son los sitios de redes sociales (SNS) en el ámbito educativo ya que los flujos de comunicación, interacción y cooperación académica implantan un escenario en el que todos se relaciona entre si permanentemente, estimulando al estudiante formar parte de un entorno que acostumbra utilizar frecuentemente, y así aprovechar que los SNS sea un recurso viable en el ámbito educativo, logrando satisfacer los interés académicos y por ende la adquisición de conocimientos.

En varios casos la forma tradicional de impartir catedra ha permitido la exclusión de la tecnología en el aula de clase, dando lugar a alternativas nuevas e innovadoras que permitan propiciar un aprendizaje significativo. El objetivo del estudio pretende determinar el uso y aplicación de las TIC en el aula y su influencia en el proceso enseñanza aprendizaje desde la percepción de los estudiantes y docentes universitarios de pregrado que cursan la asignatura de bioestadística de la Universidad Nacional de Chimborazo, Facultad de Ciencias de la Salud, Carrera de Medicina.

\section{Revisión de la literatura}

Las TIC han alcanzado a ser uno de los pilares básicos de la sociedad y es obligatorio facilitar esta cultura informática al estudiante dotando de una educación que tenga en cuenta esta realidad, entendiendo como se genera, almacena, transforma, transmite y se accede a la información en sus múltiples manifestaciones.

Tomando como base la experiencia de las comunidades europeas plasmadas en el libro blanco sobre educación y la formación, donde se da especial énfasis en la formación con miras a la incursión y mejoramiento del empleo y la competitividad fijando como prioridad la adquisición de nuevos conocimientos, tales acuerdos tienen además como finalidad acercar la escuela a la empresa, luchar contra la exclusión, conceder la misma importancia a la inversión en 
equipamiento que a la inversión en formación, en este sentido se aprecia clara e indiscutiblemente la importancia que le dan a esta temática y los resultados obtenidos (Europeas, 1995), en tal virtud es menester el tomar ejemplos de esta naturaleza y ponerlas en marcha especialmente en el sistema educativo ecuatoriano fomentando una serie de transformaciones y cambios que le permitan insertarse dentro del mundo actual y competitivo de la información y la comunicación.

Ecuador se encuentra enmarcado en el camino de la tecnología y los avances en las comunicaciones como un mecanismo de modernización del país, es así que la Secretaria Nacional de Ciencia y Tecnologia (SENACYT) se pronuncia en el apartado 3. Políticas Prioritarias, 3.4 Energía, diversificación y alternativas renovable donde expone: "Se articulará y promoverán la vinculación, cooperación e integración del Ecuador a las redes mundiales de la información, implementando redes ciudadanas donde se determinen las necesidades y se desarrollen soluciones científico-tecnológicas en campos, como: infraestructura para el acceso, teleducación, tele salud, gobierno en línea y comercio electrónico, involucrando a la sociedad civil, al sector privado y al sector público". La corriente tecnológica científica demanda cada vez más de universidades competentes, actualizadas en el uso de las TIC, esto será posible cuando se integre las TIC en el currículo universitario.

\section{El modelo constructivista}

El constructivismo tiene sus raíces en la filosofía, psicología, sociología y educación, el verbo construir proviene del latín con struere, que significa arreglar o dar estructura. Su principio se basa en que el aprendizaje humano se construye, que la mente de las personas elabora nuevos conocimientos a partir de la base de enseñanzas anteriores.

Según Piaget, (1995) manifiesta que: los esquemas son modelos mentales que almacenamos en nuestras mentes. Estos esquemas van cambiando, agrandándose y volviéndose más sofisticados a través de dos procesos complementarios: la asimilación y el alojamiento. Según la teoría constructivista de Piaget, (1978) existen dos principios en el proceso de enseñanza y aprendizaje: el aprendizaje como un proceso activo, y el aprendizaje completo, autentico y real.

\section{El aprendizaje como un proceso activo}

En el proceso de alojamiento y asimilación de la información, resultan vitales, la experiencia directa, las equivocaciones y la búsqueda de soluciones. La manera en la que se presenta la información es de suma importancia. Cuando la información es introducida como una forma de respuesta para solucionar un problema, funciona como una herramienta, no como un hecho arbitrario y solitario.

\section{El aprendizaje: completo, autentico y real}

El significado es construido en la manera en que el individuo interactúa de forma significativa con el mundo que le rodea. Esto significa que se debe enfatizar en menor grado los ejercicios de habilidades solitarias que intentan enseñar una lección. Los estudiantes que se encuentran en aulas diseñadas con este método llegan aprender estas lecciones, pero les resulta más fácil el aprendizaje si al mismo tiempo se encuentran comprometidos con actividades significativas que ejemplifiquen lo que se desea aprender. Según esta teoría, a los estudiantes se 
les debe hacer hincapié en el aula en actividades completas, auténticas que resulten intrínsecamente interesantes y significativas para el alumno, actividades reales que den como resultado algo de más valor que una puntuación en un examen.

Para Vygotsky, (1978) El constructivismo social tiene como premisa que cada función en el desarrollo cultural de las personas aparece doblemente: iniciando a nivel social, y más tarde a nivel individual; al inicio, entre un grupo de personas (inter-psicológico) y luego dentro de sí mismo (intrapsicológico). Esto se aplica tanto en la atención voluntaria, como en la memoria lógica y en la formación de los conceptos. Todas las funciones superiores se originan con la relación actual entre los individuos (Valentin, Richard, Flores Argumedo, Cosios, \& Elza, 2015).

Según Ausubel (1963) el nuevo conocimiento interacciona con el conocimiento previo y, en cierta forma, se ancla en él, a través de esta interacción es como el significado lógico de los materiales educativos se transforma en significado psicológico para el aprendiz. Es lo que se conoce como principio del aprendizaje y se halla vinculado con el aprendizaje significativo (Corica, 2009).

\section{El proceso de aprendizaje de la estadística}

La estadística moderna surge de la confluencia de dos disciplinas que evolucionaron de manera independiente: la aritmética de estado (estadística) y el cálculo de probabilidades. La mayoría de las civilizaciones antiguas recogían datos sobre los impuestos recaudados, el número de soldados reclutados, bajas en batallas, censos, etc. En el siglo XVII John Graunt fue el primero en realizar tablas de mortalidad y estudios demográficos. Achenwald fue el primero en denominar Estadística, a la hasta entonces denominada aritmética de estado. El cálculo de probabilidades se desarrolló debido a los juegos de azar. En los siglos XVI y XVII hombres de ciencia tan importantes como Galileo, Fermat y Pascal, dedicaron mucho tiempo al desarrollo del cálculo de probabilidades con objeto de resolver problemas planteados por los juegos de azar. La fórmula clásica del cálculo de probabilidades "la probabilidad de que ocurra un determinado suceso es igual al número de casos favorables al suceso, dividido por él número de casos totales que pueda ocurrir" es debido a Pascal.

En los siglos XVII y XVIII Newton revolucionó la ciencia e introdujo junto a Leibnitz el cálculo infinitesimal y de modelización matemática de los fenómenos físicos, como consecuencia de ello se puso de manifiesto la necesidad de la exactitud de las medidas y el desarrollo de cálculos de errores. En el siglo XVIII De Moivre comprobó que la distribución binomial podía aproximarse a la normal cuando el número de casos era grande. Este autor junto a Laplace fueron de los primeros en aplicar el cálculo de probabilidades a los datos demográficos, contribuyendo a unificar la estadística y el cálculo de probabilidades en una sola disciplina. Una figura muy importante en el campo del cálculo de probabilidades, aunque paso desapercibido en su tiempo, fue Bayes, autor del teorema que lleva su nombre.

En el siglo XIX una de las figuras más relevantes en el campo de la física y de la estadística fue Gauss. Este científico hizo magníficos estudios sobre la curva normal, a la cual también se la conoce como curva de Gauss, aunque ya era conocida antes de que éste importante matemático hiciera sus estudios, también realizo profundos análisis sobre la teoría de 
errores, y desde el punto de vista práctico la aplicó a la astronomía. Otras figuras relevantes del siglo XIX en el campo de la estadística fueron Newcomb, que realizo importantes estudios en relación a la estimación de parámetros, y K. Pearson, que trabajo, entre otros temas, sobre correlación y regresión entre variables.

En el siglo XX, junto a Pearson, que hizo sus trabajos entre el siglo XIX y XX, el autor más importante fue Fisher, el cual dedico gran parte de su tiempo al análisis de modelos matemáticos multivariantes; entre otras aportaciones invento el análisis discriminante. Es Pearson, Wald y Neyman quienes desarrollaron la teoría del contraste de hipótesis. A partir de 1970 la estadística ha cobrado una gran dimensión, entre otras razones por la generalización del uso de los computadores, lo que ha permitido utilizar técnicas estadísticas que, aunque conocidas desde hace tiempo, se aplicaban en pocas ocasiones debido a la dificultad de los cálculos.

En la actualidad la estadística es una disciplina que está evolucionando; la sociología, la psicología, la utilización creciente de encuestas, la generación del control de calidad, la aplicación a las ciencias de la salud, las nuevas teorías físicas, etc., son algunas de las razones que han motivado una gran demanda de estudios. Dentro del campo de la estadística aplicado a las ciencias de la salud como los problemas biomédicos J. Graunt realizó las primeras tablas de mortalidad conocidas. Galton primo de Darwin es considerado el padre de la bioestadística, el cual junto a K Pearson y Fisher establecieron las bases de la bioestadística moderna (Alvarez Caceres, 2007).

La estadística como rama de la matemática aplicada, su uso se manifiesta en la recopilación, procesamiento y análisis de la información relacionada con los datos económicos, políticos sociales, biológicos, psicológicos, físicos, químicos, entre otros (Espindola Artola, Gutiérrez Alvarez, Castellanos Pupo, Yordi González, \& Miranda Carbonell, 2012).

El empleo de la estadística en ciencias de la salud es fundamental desde la perspectiva epidemiológica, ya que propicia el estudio de enfermedades, sin embargo, las dificultades relacionadas con el aprendizaje de esta asignatura dan lugar a insuficiencias en la asimilación de contenidos por parte de los estudiantes (Claveria Sánchez et al., 2017).

\section{Las TIC en la educación}

Las TIC han evolucionado espectacularmente en especial por la interconexión a través de la internet, lo que ha impacto en el ámbito educativo el mismo que debe adaptarse a esta revolución digital y adecuarse para su utilización didáctica conociendo sus límites y peligros que las TIC plantea a la educación y reflexionar sobre el nuevo modelo de sociedad que surge de esta tecnología y sus consecuencias. Las TIC en el aula ya son una realidad y la responsabilidad de los actores en el proceso enseñanza aprendizaje es introducir estos cambios que demandan la evolución tecnológica en la actualidad, ofreciendo una educación de calidad, mejorando los procesos y logrando aprendizajes significativos en los estudiantes.

El sistema educativo no puede quedar al margen de los nuevos cambios debe atender a la formación de los nuevos ciudadanos y la incorporación de las nuevas tecnologías ha de hacerse con la perspectiva de favorecer los aprendizajes y facilitar los medios que sustenten el desarrollo 
de los conocimientos y de las competencias necesarias para la inserción laboral y profesional de calidad evitando la brecha digital que genere capas de marginación como resultado de la analfabetización digital.

El software educativo en la dinámica del proceso enseñanza aprendizaje de la estadística se convierte en una herramienta de apoyo para a la apropiación socializada e intencional en el aula de la cultura estadística, que permitirá la transformación sostenible del proceso enseñanza aprendizaje de la asignatura (Faustino \& Luis, 2013).

En la misma línea, investigadores como Rojas (2001), Márquez (2004), Pardo, Izquierdo, \& Fuentes (2004), Pérez \& Marrero (2005), Valdés Tamayo (2007), Ruíz Ortega (2007), Faustino, Pérez, \& Diéguez (2012) mencionan la utilización de medios didácticos tecnológicos en el proceso enseñanza aprendizaje de la estadística. Sin embargo la variabilidad de las funciones atribuidas por los docentes a dichos recursos tecnológicos es insuficiente, y en la mayoría de los casos, son utilizados para funciones relacionadas con la transmisión de información y como un recurso del currículo educativo. Una vez analizada la literatura, el alcance de la investigación es descriptiva, correlacional y explicativa

Se plantea la hipótesis: El nivel de enseñanza y aprendizaje de la bioestadística en los alumnos de tercer semestre de la Universidad Nacional de Chimborazo, Facultad de Ciencias de la Salud, obtendrán mejores resultados con la aplicación de las TIC en el aula.

- Variable Independiente: Uso y aplicación de las TIC en el aula (Causa)

- Variable dependiente: Enseñanza aprendizaje de la bioestadística (Efecto)

\section{Metodología}

La presente investigación es de tipo documental y de campo con un enfoque CuantiCualitativo (Mixto) con un diseño o método no experimental ex post facto retrospectivos, transversal - descriptivo Correlacional - causal, además para su realización y comprobación se incluirán cifras y estadísticas al ser evaluados los resultados. Se trabaja con todo el universo o población que estuvo constituida por 82 estudiantes que toman la asignatura de bioestadística, y 3 Docentes que dictan dicha catedra a los estudiantes de la carrera de medicina en el periodo Octubre 2016 Marzo 2017.

La técnica empleada para la recolección de los datos fue la encuesta, y el instrumento el cuestionario que está conformada de 27 preguntas cerradas, aplicando la escala tipo Lickert. A su vez se empleó el método de consistencia interna basado en el coeficiente del Alpha de Crombach que permite estimar la fiabilidad de un instrumento de medida a través de un conjunto de ítems, en el estudio la confiabilidad del cuestionario fue satisfactorio. Con los datos recolectados se procede a la codificación que fueron transformados a símbolos numéricos para poder ser contados y tabulados.

Los datos obtenidos de las encuestas se procesaron utilizando una base de datos en el software Statistical Package for the Social Sciences (SPSS) Versión 23, los resultados son analizados utilizando estadígrafos descriptivos e inferenciales. Se calcularon distribuciones de frecuencias y porcentajes 


\section{Resultados}

La muestra poblacional estuvo formada por un total de 82 estudiantes y 3 docentes que imparten catedra de bioestadística. Es así que en primer lugar se analizó las preguntas del cuestionario aplicando el coeficiente del Alpha de Cronbach para determinar su confiabilidad, es así que sus valores tanto de estudiantes como de docentes es muy cercano a la unidad lo que representa un nivel satisfactorio y el instrumento es fiable.

Tabla 1. Coeficiente del Alpha de Cronbach

\begin{tabular}{|c|c|c|c|}
\hline & Alfa de Cronbach & $\begin{array}{l}\text { Alfa de Cronbach basada en los elementos } \\
\text { tipificados }\end{array}$ & $\mathrm{N}$ de Elementos \\
\hline Estudiantes & ,990 & ,992 & 27 \\
\hline Docentes & ,978 & ,992 & 27 \\
\hline
\end{tabular}

El mayor porcentaje tanto docentes $100 \%$ como estudiantes $54 \%$ disponen del computador en sus hogares para cumplir con sus tareas educativas, sin embargo es importante conocer si esta herramienta tecnológica posee características técnicas actualizadas y de punta ya que existe variadas aplicaciones que influirán en el desarrollo académico, siendo los resultados que el $32 \%$ de los estudiantes consideran que su equipo está dotado a gran medida de tecnología de punta, en el caso de los docentes que consideran que el $33 \%$ tiene un equipo de tecnología de punta frente al $67 \%$ que considera a regular medida su equipo, en lo referente a que si la impresora es una herramienta primordial para cumplir con su labor educativa consideran tanto estudiantes como docentes que el $66 \%$ es primordial.

En lo referente a la incorporación de recursos tecnológicos en el aula de clase como es el proyector se demuestra claramente por parte de los estudiantes que en el $49 \%$ nunca los usa y casi nunca en el $12 \%$, respuesta similar se obtiene del docente es así que $33 \%$ respondieron algunas veces, casi nunca $33 \%$, y nunca $33 \%$, como se demuestra en el gráfico 1

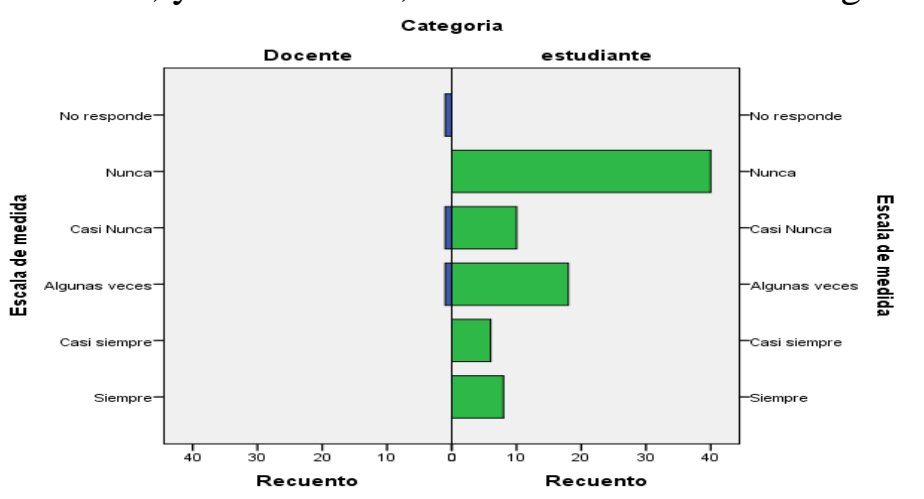

Gráfico 1. Uso de las TIC en el aula por parte de los docentes

En el proceso enseñanza aprendizaje el docente incorpora recursos en el aula como un medio de comunicación y despeje de dudas como son los foros metodológicos, temático y científicos?, una vez obtenido la información de cada uno de los foros tanto del docente como de los estudiantes esta información es variada, se desarrolló un promedio de los foros desde la percepción de los estudiantes y docentes es así que afirman los estudiantes que solamente el $1 \%$ siempre lo usan, el $20 \%$ casi siempre, $34 \%$ Algunas veces, $25 \%$ Casi nunca y el $17 \%$ Nunca, información que varía con lo que opina los docentes siendo el $33 \%$ opina que siempre lo usan, frente al $45 \%$ que casi siempre y el $22 \%$ lo usa algunas veces, esta situación se da ya que los 
docentes aplican foros en su actividad académica sin embargo los estudiantes no la utilizan como se demuestra en la tabla 2 y gráfico 2.

Tabla 2. Utilización de foros en el proceso enseñanza aprendizaje

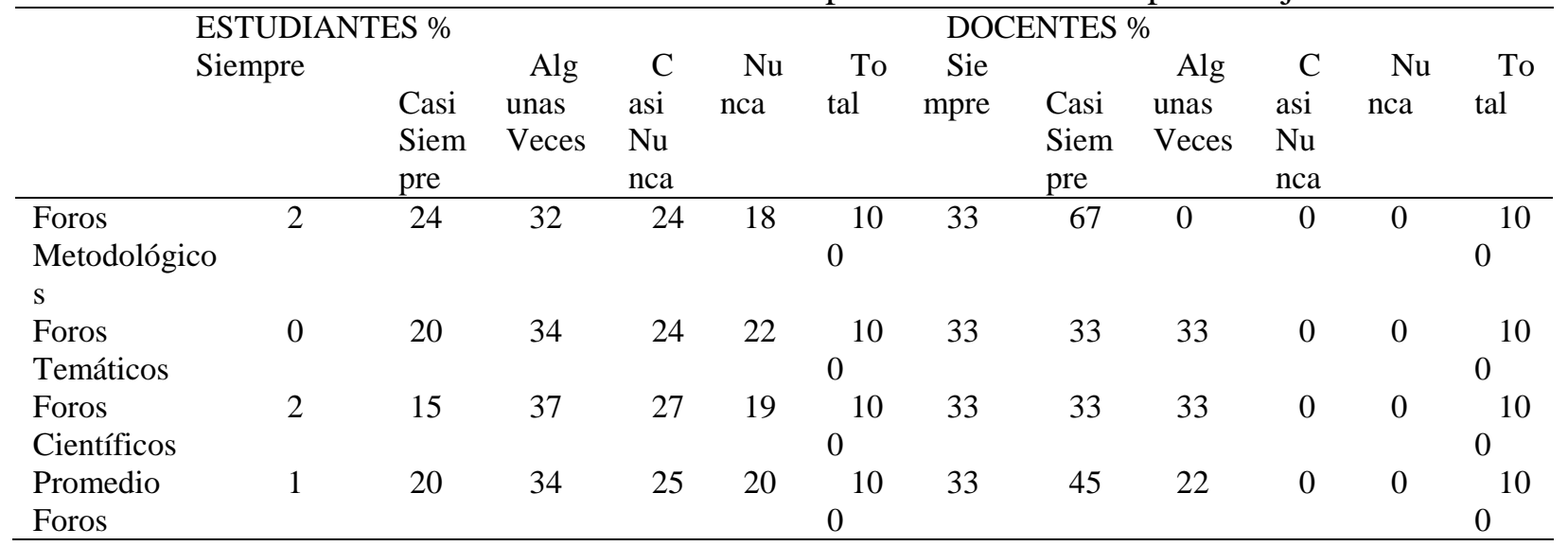
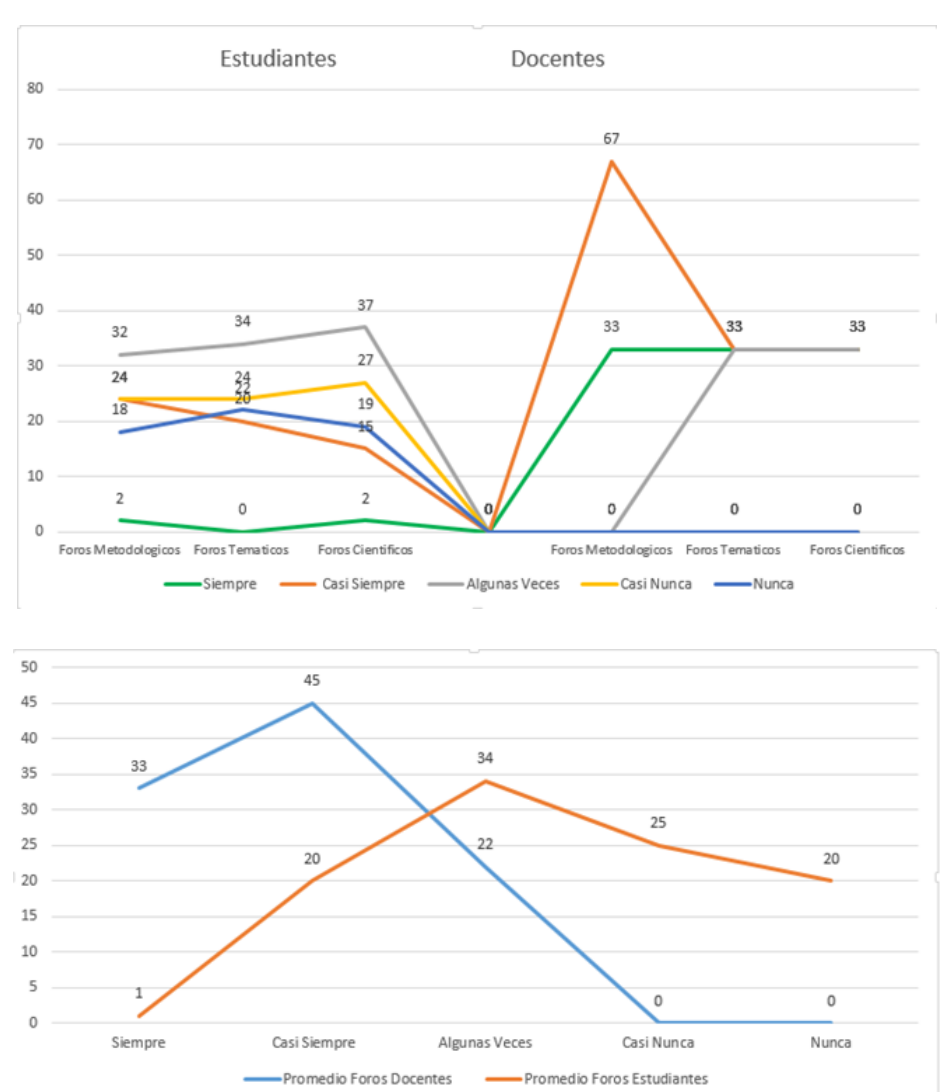

Gráfico 2. Uso de los Foros desde la perspectiva de los estudiantes y docentes

La internet es un recurso tecnológico que los estudiantes tienen acceso en el $51 \%$ desde su domicilio y desde otros sitios en un $100 \%$, a su vez consideran que la información que obtiene de la misma es segura y confiable en un $58 \%$, los docentes tienen acceso a la internet desde su domicilio en el 100 \%. La comunicación, interacción y colaboración que presta los recursos TIC mediados por la internet y que se usa como apoyo en el proceso formativo como 
los chat, videoconferencia, Messenger, correo electrónico y blogs, según los datos procesados se afirma que el chat, el correo electrónico y el Messenger son los medios más utilizados por los estudiantes, frente al poco o casi nada de uso de estos recursos por parte de los docentes, siendo solo el correo electrónico el que más es utilizado por los mismos que es representado en el gráfico 3 .

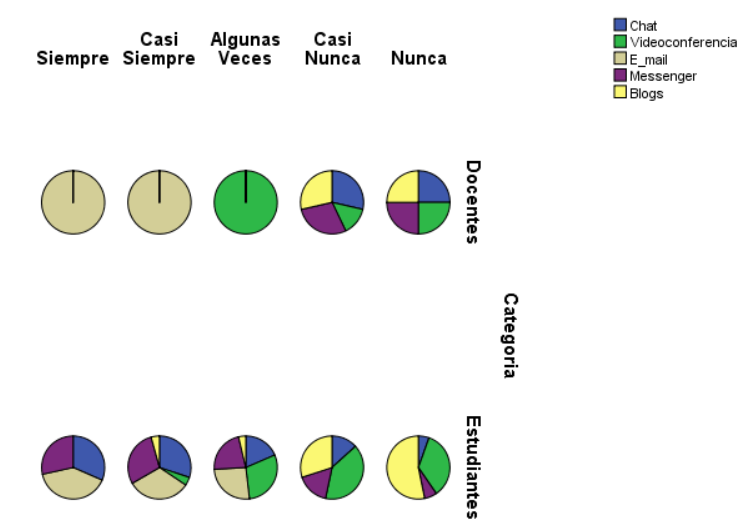

Gráfico 3. Uso de recurso de comunicación, interacción y colaboración utilizados por estudiantes y docentes

Los sistemas de gestión de aprendizaje en el caso de estudio se analizó si los docentes utilizan las aulas virtuales como una alternativa moderna , contribuyendo a un aprendizaje cooperativo y siendo un complemento de la educación presencial y en función a las respuestas proporcionadas por los docentes solamente el $33 \%$ lo usan frente al $67 \%$ que no lo usan, de la misma manera se investigó el uso de software educativo que facilite el proceso enseñanza aprendizaje lo que respondieron su uso en un $67 \%$, y en un $33 \%$ no lo usa.

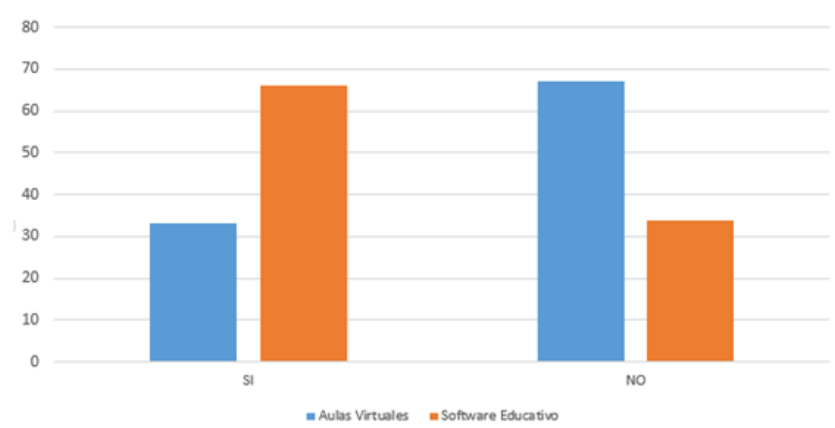

Gráfico 4. Uso de aulas virtuales y software educativo por parte de los docentes

La internet es la red de información más amplia en el mundo, consecuentemente es de gran ayuda en las tareas de investigación y desarrollo educativo por lo tanto los estudiantes como los docentes afirman que facilita el acceso a información y al desarrollo de nuevos conocimientos, con respecto a la ofimática se obtuvo información en donde el Word y Power Point son las aplicaciones más usadas y ayudan al desarrollo de trabajos y presentaciones, a su vez los docentes creen firmemente que las aulas virtuales, el software educativo, la internet y sus diferentes recursos ayudaran en el desarrollo formativo facilitando el proceso enseñanza aprendizaje. 


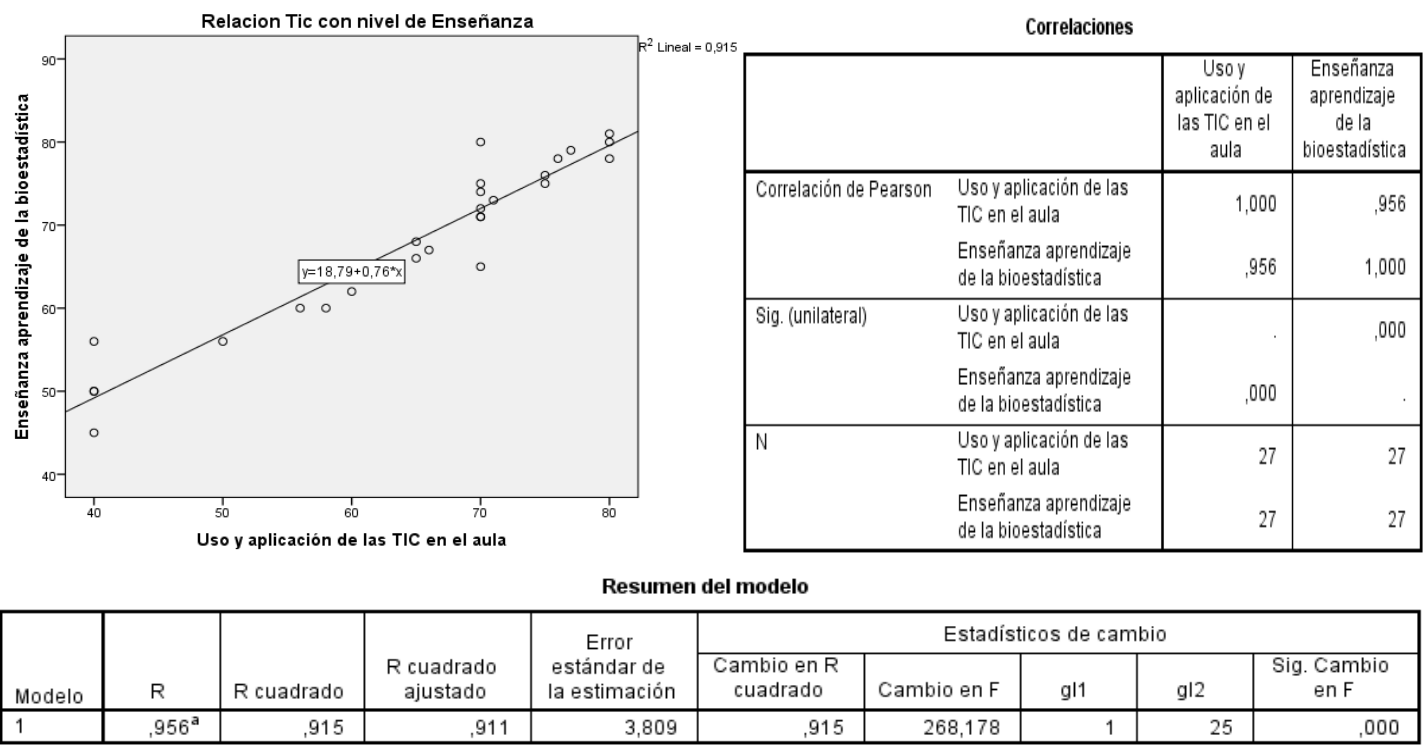

a. Predictores: (Constante), Enseñanza aprendizaje de la bioestadística

Gráfico 5. Análisis de correlación (Uso y aplicación de las TIC en el aula - nivel de Enseñanza aprendizaje de la bioestadística)

Se realizó el análisis de correlación entre las variables el uso de las TIC con el nivel de enseñanza lo que se visualiza una correlación positiva muy fuerte (0 .956), este dato implica que las TIC influyen directamente en un $91 \%$ en el en el proceso enseñanza aprendizaje de la bioestadística comprobándose la aceptación de la hipótesis planteada

\section{Discusión}

En función a la búsqueda de determinar el impacto del uso y aplicación de las Tics en el proceso enseñanza aprendizaje de la bioestadística en una media del $73 \%$ coinciden en las bondades y beneficios que aportan las TIC en el proceso educativo, sin embargo, el $81 \%$ manifiestan que no se utilizan en el Aula.

Reátegui, en su trabajo, que indica que la actitud de los docentes del Departamento de pediatría de la UNMSM hacia las TIC fue para el $86 \%$ favorable y muy favorable, demostrando el interés y la predisposición hacia la utilización de la tecnología de punta en provecho del proceso enseñanza aprendizaje (Américo et al., 2015).

La utilización del software educativo y sistemas de gestión de aprendizaje como las aulas virtuales facilitan la enseñanza y aprendizaje de la bioestadística estos recursos educativos ayudan a un aprendizaje significativo por descubrimiento capases de desarrollar habilidades cognitivas en el estudiante, la utilización de las aulas virtuales en un ambiente educativo complementa el aprendizaje presencial y da lugar a tener una interacción de manera ubicua con el estudiante facilitando compartir recursos educativos entre el docente y estudiante, estos recursos muy importantes y necesarios que se debería incluir en gran escala y en todas las asignaturas en el proceso enseñanza aprendizaje.

López observó que la mayoría de los docentes (77.3\%) niegan que las TIC desplacen al docente en su labor académica. Esta perspectiva sobre la importancia de la acción docente es 
fundamental para la aceptación de la tecnología, la cual no disminuye ni demerita la función del docente (López \& Chávez, 2013).

Los docentes en la actualidad deben estar en la capacidad de usar la tecnología para crear ambientes de aprendizaje, innovadores, donde realmente se pueda construir conocimientos mediados por las TIC donde el docente solo sea el guía y el estudiante sea el actor principal en el proceso enseñanza aprendizaje. Caicedo por su parte también evidenció que los profesores tienen creencias positivas hacia el uso de las TIC en la educación, en tanto creen que pueden apoyar los procesos de enseñanza y de aprendizaje (Caicedo Tamayo \& Rojas Ospina, 2014).

\section{Conclusiones}

La investigación da a conocer el grado de la utilización de las TIC en el proceso enseñanza aprendizaje en el aula por parte de los docentes que imparte la catedra de Bioestadística de la carrera de Medicina de la Universidad Nacional de Chimborazo. Los resultados extraídos han dado a conocer un bajo índice de integración de las TIC en el aula que ayude en el proceso educativo.

En la bioestadística, es poco frecuente la utilización del computador, pizarrón electrónico, software educativo y más recursos TIC donde los estudiantes puedan realizar cualquier análisis estadístico orientado por el docente quien es el actor principal que fomente y motive el aprendizaje y desarrollo de habilidades cognitivas, la generación de ideas intuitivas a través del ensayo y error donde se genera nuevo conocimiento.

La percepción generalizada de los estudiantes a cerca de las TICs aplicadas en el Aula es inexistentes, tampoco se utilizan recursos de comunicación e interacción para el intercambio de conocimiento entre los diferentes actores educativos.

Los docentes escasamente utilizan recursos didácticos multimedios, aulas virtuales, la utilización de estrategias como el seminario el foro, taller son limitadas en el proceso enseñanza y aprendizaje. Los docentes están de acuerdo que las TIC son herramientas que logran aprendizajes significativos y su aplicación beneficiaria en el proceso enseñanza aprendizaje, para que de esta manera se oriente a una metodología didáctica.

La mayoría de los docentes utilizan el internet y sus recursos para la consulta de información, no existe un uso adecuado de las redes sociales en las aulas de clase en el proceso enseñanza aprendizaje. De igual forma, un gran porcentaje, tanto de docentes como estudiantes que participaron en el estudio, han manifestado que el uso de las TIC contribuirá en el aumento del interés por las temáticas de la asignatura permitiendo que los estudiantes adquieran la información que el docente imparte en menor tiempo y con claridad, logrando mejorar el rendimiento académico de los estudiantes comprobándose mediante la estadística la hipótesis planteada.

\section{Recomendaciones}

El docente como formador está bajo su responsabilidad la enseñanza de procedimientos y destrezas que permitan el manejo y aplicación de los contenidos estadísticos, con la utilización de herramientas tecnológicas que fortalezcan un proceso enseñanza aprendizaje motivador, dinámico y eficaz. 
Implementar planes de formación del profesorado, que consideren metodologías con respecto a la utilización de las TICs en el aula, reforzando al máximo el aprovechamiento de los recursos educativos tecnológicos que la universidad ofrece a los docentes y estudiantes desarrollando competencias en este uso, tales como la plataforma Moodle Aulas virtuales, Laboratorios, Simuladores, Software multimedio, etc. Contar con profesionales de áreas técnicas, gráficas y pedagógicas que apoyen a los docentes en el diseño, implementación de estos recursos TIC en el aula de clase.

Para que las TIC favorezcan el cambio pedagógico, se requiere que se base en metodologías de enseñanza centradas en el estudiante, que favorezcan cambios de roles en docentes y estudiantes con el uso que potencia las TIC como espacios donde se comparta, distribuya y se de una construcción colectiva de conocimiento, disminuyendo la brecha digital.

\section{Trabajos Futuros}

De la misma manera se considera controlar las variables del contexto como porcentaje de apoyo de las TIC en el curso y el tipo de recurso utilizado, con el fin de obtener una medición del impacto aún más objetiva, así mismo es imperiosa la necesidad de conocer el motivo por el cual los docentes no utilizan las TIC en el proceso enseñanza aprendizaje dentro del aula y buscar estrategias para ubicar el uso de estas herramientas tecnológicas de manera indispensable para la flexibilidad curricular y académico.

\section{Bibliografía}

Alvarez Caceres, R. (2007). Estadistica aplicada a las ciencias de la salud.

Américo, L., Guzmán, R., Hernán, M., Sotomayor, I., Ramírez, M., Susana, R., ... San, M. De. (2015). Actitud de estudiantes y profesores del Departamento de Pediatría hacia las tecnologías de la información y comunicación ( TICs ) Attitude of students and teachers of the Department of Pediatrics towards information. Scielo.org.pe, 76(3), 261-264. Retrieved from http://www.scielo.org.pe/pdf/afm/v76n3/a07v76n3.pdf

Cabero Almenara, J. (1996). Nuevas Tecnologias, Comunicacion y Educación.

Caicedo Tamayo, A. M., \& Rojas Ospina, T. (2014). Creencias, conocimientos y usos de las TIC de los profesores universitarios. Educación $Y$ Educadores, 17(3), 517-533. https://doi.org/10.5294/edu.2014.17.3.7

Claveria Sánchez, G., Ríos Ruiz, Y., Molina Herrera, M., Sosa Cabrera, I., Pérez Arruti, A., \& Ramona, C. P. (2017). Actividades de aprendizaje sobre estadistica descriptiva dirigidas a estudiantes del Tecnico Medio en Vigilancia y Lucha Antivectorial. Tecnosalud. Retrieved from http://www.tecnosaludcmw2017.sld.cu/index.php/socoenf/tecnosalud2017/paper/viewFile/81/4 4

Corica, A. R. (2009). Aprender Matemática en la Universidad: la perspectiva de estudiantes de primera año. Revista Electrónica de Investigación En Educación En Ciencias, 4(1), 10-27. Retrieved from $66662009000200002 \&$ script=sci_arttext\&tlng=en

http://www.scielo.org.ar/scielo.php?pid=S1850-

Espindola Artola, A., Gutiérrez Alvarez, M., Castellanos Pupo, X., Yordi González, I., \& Miranda Carbonell, M. (2012). Estrategia didáctica para la dinámica del proceso docente educativo de la Matemática en la especialidad Bioestadística Didactic strategy for the dynamic of the teachingeducative process of Mathematics, within Biostatistics specialty, 12(2), 347-359. Retrieved from http://scielo.sld.cu/pdf/hmc/v12n2/hmc15212.pdf

Europeas, C. (1995). Libro blanco sobre la educación y la formación. Enseñar y aprender, hacia la 
sociedad cognitiva, (95), 62. Retrieved from http://eurlex.europa.eu/LexUriServ/LexUriServ.do?uri=COM:1995:0590:FIN:ES:PDF

Faustino, A., \& Luis, S. P. (2013). Utilización De Las Tic En La Enseñanza De La Estadística En La Educación Superior Angolana. (Spanish). Use Of The Ict For Statistics Teaching In The Angolan Superior Education. (English). Retrieved from http://search.ebscohost.com/login.aspx?direct=true\&db=fua\&AN=94767945\&lang=es\&site=eh ost-live

FAUSTINO, A., PÉREZ, N., \& DIÉGUEZ, R. (2012). Propuesta didáctica para el proceso de formación del pensamiento lógico matemático complejo en la educación superior angolana. Revista Pedagogía Profesional V, (10). Retrieved from http://www.redalyc.org/pdf/3537/353744535001.pdf

Hidalgo Cajo, B. G., Hidalgo Cajo, D. P., \& Hidalgo Cajo, I. M. (2017). El impacto de las redes sociales como herramientas de comunicación, interacción y colaboración en el proceso enseñanza aprendizaje en la educación superior. SATHIRI, 12(104-113).

López, M., \& Chávez, J. (2013). La formación de profesores universitarios en la aplicación de las TIC. Sinéctica, $41, \quad 2-18 . \quad$ Retrieved from http://www.scielo.org.mx/scielo.php?script=sci_arttext\&pid=S1665109X2013000200005\&lng=es\&nrm=iso\&tlng=es

MÁRQUEZ, J. C. (2004). Una revisión de las evidencias de fiabilidad y validez de los cuestionarios de actitudes y ansiedad hacia la estadística. Statistics Education Research Journal, 3(1), 5-28.

Pardo, M. E., Izquierdo, J., \& Fuentes, H. (2004). Las tecnologías de la información y las comunicaciones en la dinámica del proceso docente educativo en la educación superior. Tesis presentada en opción al grado científico de doctor en ciencias pedagógicas. Universidad de Oriente, Centro de estudios de educación superior" Manuel F. Gran”, Santiago de Cuba.

PÉREZ, S., \& MARRERO, S. (2005). La computación como ciencia aplicada. La automatización de la clave dicotómica para la clasificación de familias Botánicas, con fines didácticos. La Habana, Cuba. Editorial Pueblo Y Educación, 18(2), 13-15.

Rojas, A. R. (2001). Introducción al estudio de los medios de enseñanza. La Habana: Centro de Estudios Para El Perfeccionamiento de La Educación Superior (CEPES).

Ruiz, J. C., Herrera, V., Herdoiza, I., \& Diaz, C. (2010). Secretaria Nacional de Ciencia y Tecnologia de Ecuador. Retrieved from http://repositorio.educacionsuperior.gob.ec/bitstream/28000/913/1/L-SENESCYT-0034.pdf

Ruíz Ortega, F. J. (2007). Modelos didácticos para la enseñanza de las ciencias naturales. Revista Latinoamericana de Estudios Educativos (Colombia), 3(2).

Valdés Tamayo, P. R. (2007). Libros electrónicos multimedia para el estudio independiente en la semipresencialidad. Ciudad de Las Tunas: Editorial Universitaria, 2007.

Valentin, C., Richard, Y., Flores Argumedo, J. C., Cosios, V., \& Elza, N. (2015). La plataforma Moodle y su influencia en el aprendizaje del área de educación para el trabajo de los estudiantes de $5^{\circ}$ grado de la Institución Educativa CNV Vitarte-UGEL $\mathrm{N}^{\mathrm{o}}$ 06-2015. Retrieved from http://repositorio.une.edu.pe/handle/UNE/1154 